\title{
Performance of Multiple Pulse Multiple Delay modulated UWB Signals in a Multiple Access Indoor Wireless Channel (U)
}

F. Nekoogar, F. U. Dowla

"2003 IEEE Pacific Rim Conference on Communications, Computers, and Signal Processing," Victoria, B.C. Canada, August 28, 2003

U.S. Department of Energy"

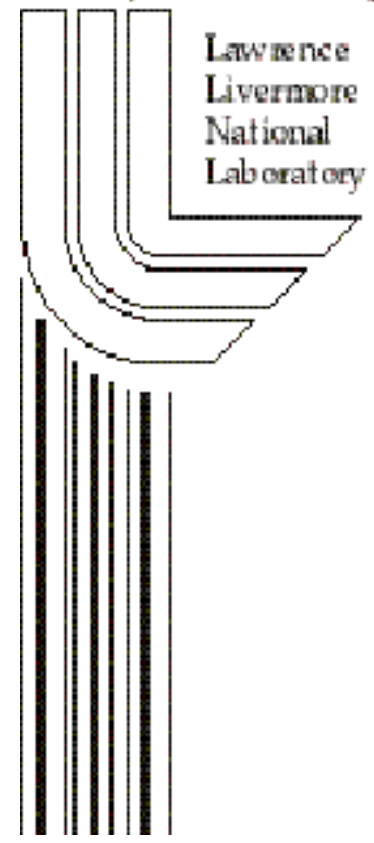

June 12, 2003 


\section{Disclaimer}

This document was prepared as an account of work sponsored by an agency of the United States Government. Neither the United States Government nor the University of California nor any of their employees, makes any warranty, express or implied, or assumes any legal liability or responsibility for the accuracy, completeness, or usefulness of any information, apparatus, product, or process disclosed, or represents that its use would not infringe privately owned rights. Reference herein to any specific commercial product, process, or service by trade name, trademark, manufacturer, or otherwise, does not necessarily constitute or imply its endorsement, recommendation, or favoring by the United States Government or the University of California. The views and opinions of authors expressed herein do not necessarily state or reflect those of the United States Government or the University of California, and shall not be used for advertising or product endorsement purposes. 


\title{
Performance of Multiple Pulse Multiple Delay Modulated UWB Signals in a Multiple Access Indoor Wireless Channel
}

\author{
Faranak Nekoogar and Farid Dowla \\ Department of Applied Science, University of California Davis \\ Lawrence Livermore National Laboratory
}

E-mail:

\{nekoogar1, dowla1\}@1lnl.gov

\begin{abstract}
In this paper, the performance of a two user UWB multiple access (UWB-MA) system based on multiple-pulse multiple-delay (MPMD) modulation scheme in an indoor wireless channel is evaluated by computer simulations. The indoor multipath propagation channel model used in this study is based on the modified statistical Saleh-Valenzuela model proposed by Foerester and $\mathrm{Li}$ from Intel. The simulation results indicate that the multipath performance of MPMD modulated signals in a multiple access system outperforms the nonmultipath case as the number of autocorrelation function (ACF) sampling points increases for each user. This is an unusual but important result, since MPMD receiver exploits multipath phenomenon in indoor wireless channels to increase the BER performance, hence the transmission rate in a UWB-MA system.
\end{abstract}

\section{INTRODUCTION}

Ultra-wideband systems modulate carrierless, short duration pulses with fractional bandwidths larger than 20\% [1]. The short duration pulses provide fine delay resolution properties and make UWB systems good candidates for indoor wireless communication channels in short range high data rate applications such as WPANs. These applications require that multiple transmitters coexist inside offices and residential buildings where the indoor wireless channel introduces a dense multipath environment. Therefore, there is a need for proper channelization techniques to separate multiple users in wireless indoor channels. The conventional modulation schemes used in UWB multiple access systems such as Pulse Amplitude Modulation (PAM) or Pulse Position Modulation (PPM) employ the same pulse shape and modulate the transmit pulse based on changing amplitude or position, of the pulses respectively. Both of these modulation methods require stringent synchronization and do not perform well in dense multipath environments without the additional help form rake

This work was performed under the auspices of the U.S. Department of Energy by University of California Lawrence Livermore National Laboratory under contract No. W-7405-Eng-48. receivers. Another concern with PPM/PAM modulation techniques is that the multiple access interference (MAI) increases with the number of users. This is due to increased cross-correlation between similar pulses of multiple channels, raising thus the noise floor in such systems.

A recently proposed modulation scheme called Transmitted-Reference (TR) is defined as the transmission of a pair of pulses separated by a unique delay for each channel to represent data bits $[7,8]$. This method has the advantage of sending the same pulse twice through an unknown channel where both pulses are distorted the same way and detection becomes easier with an autocorrelation receiver without the stringent synchronization requirements that exist in conventional approaches. Although this technique performs well in multipath environments and addresses the synchronization problem, the MAI concern still exists since a single UWB pulse shape is used for all channels. In order to reduce the MAI effect, delays used between the pair of pulses in TR modulated signals have to be relatively large from one user to another.

Multiple-pulse multiple-delay (MPMD) technique addresses MAI problem by using mutually orthogonal TR modulated pulses for multiple users. The combination of orthogonal pulse shape coding and delay coding (TR), results in high bit error rate performance and increased channel capacity in UWB systems [5,6]. The use of multiple uncorrelated pulses in MPMD modulation alleviates the time delay restrictions that exist in original TR modulation scheme where a single pulse shape is used for all channels. In this method, the time delays used to separate the pair of pulses in each channel can be as small as a fraction of the pulse duration for each user providing excellent BERs.

This paper studies the performance of MPMD modulated UWB pulses in a dense multipath indoor channel based on Saleh-Valenzuela model proposed by Foerester and Li. Section 2 provides a brief overview of MPMD modulation scheme. Section 3 describes the indoor channel model used for computer simulations. Section 4 provides the BER performance analysis of a MPMD receiver in a dense multipath channel followed by conclusions in section 5 . 


\section{MPMd MOdULATION OVERVIEW}

MPMD approach uses multiple mutually orthogonal TR modulated pulses to transmit the data bits. A symbol consists of two similar pulses $\left(P_{n}\right)$ separated by a delay $\left(D_{n}\right)$ where pulses and delays are unique to each user. The first pulse is fixed and called reference pulse (Ref) and the second pulse is modulated with data, called data pulse (Data). The data pulse modulation scheme is based on the polarity of the reference pulse $[5,7,8]$. Assuming a uniform symbol period, the general signal model of a MPMD system with $N$ users can be expressed as

$$
s(t)=\sum_{n=1}^{N} \sum_{m=1}^{M} \sqrt{E_{p, n}}\left[P_{n}\left(t-(m-1) T-(-1)^{b^{(}{ }^{(n)}} . P_{n}\left(t-(m-1) T-D_{n}\right)\right]\right.
$$

$N=$ Number of users, $M=$ Number of transmitted bits, $E_{p, n}=\mathrm{n}^{\text {th }}$ user's signal energy (normalized for all users), $P_{n}(t)=\mathrm{n}^{\text {th }}$ user's UWB pulse (orthogonal to other user's pulses), $b_{m}{ }^{(n)}=\left[b_{1}{ }^{(\mathrm{n})}\right.$, $\left.\ldots, \mathrm{b}_{\mathrm{M}}{ }^{(\mathrm{n})}\right] \mathrm{n}^{\text {th }}$ user's $\mathrm{m}^{\text {th }}$ data bit with $\mathrm{b}_{\mathrm{m}}{ }^{(\mathrm{n})} \in[0,1], D_{n}=\mathrm{n}^{\text {th }}$ user's delay, and $T=$ Pulse repetition period.

The pulses used in this study are short duration chirp pulses with different start and end frequencies. Chirp pulses that do not overlap in frequency band are theoretically uncorrelated with each other and can be separated using autocorrelation techniques.

MPMD receiver takes advantage of the fact that the shape of a typical UWB pulse experiences significant degradations in dense multipath channels where the shape of it's autocorrelation function (ACF) is relatively preserved at the receiver. Therefore, sampling the shape of ACF provides significant improvements to the detection process of multiple pulse systems. MPMD receiver samples the ACF of each user's pulse at both zero- and non-zero lags and matches them to the corresponding samples taken at transmitter rather than sampling and matching the pulse shape. The input signal to the receiver in an indoor channel is given by

$$
r(t)=s(t) * h(t)+w(t)
$$

Where $s(t)$ is the combined signal for $N$ users and $M$ data bits from (1), $h(t)$ is the multipath channel impulse response, and $w(t)$ is AWGN with zero mean and two-sided power spectral density $N_{0} / 2$. The ' $*$ ' notation represents the convolution operation. The multipath channel can be modeled as a linear filter and is assumed to be a set of $L$ delayed impulses with different magnitudes and signs representing different multipath components such as

$$
h(t)=\sum_{l=1}^{L} a_{l} \delta\left(t-\tau_{l}\right)
$$

$L$ represents the number of reflected signals in a multipath channel, $a_{l}$ is the amplitude of path $l, \tau_{l}$ is the random delay of each path, and $\delta(t)$ is the Dirac-delta function. Therefore, the received signal can be expressed as

$$
r(t)=\sum_{l=1}^{L} a_{l} s\left(t-\tau_{l}\right)+w(t)
$$

The MPMD receiver has two steps. Step one samples the received signal's ACF shape at multiple times. The sampling is achieved from multiplying the received signal by its multiple delayed versions. The next step matches the sampled values from the first step to the sampled values of ACFs of the original pulses. Note matching is performed over the second order statistics, such as the ACF, and not on the signal shape, as is conventionally performed in classical matched filters. Fig. 1 represents the MPMD receiver block diagram.

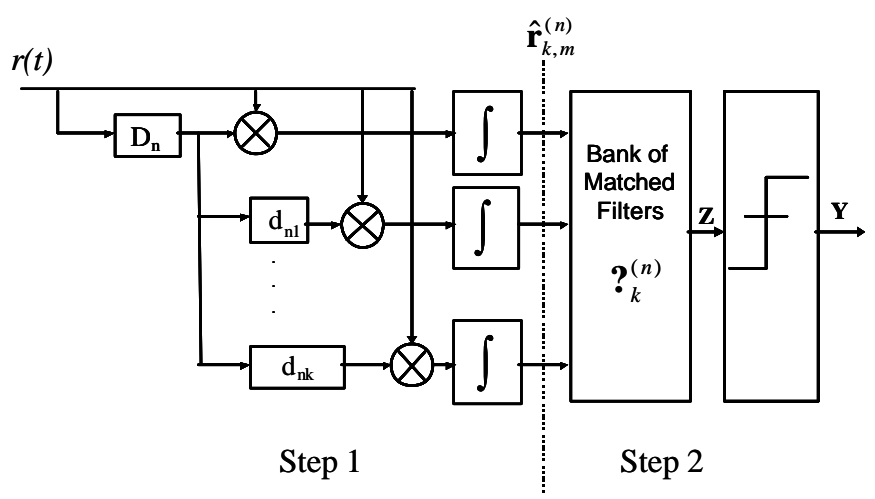

Figure 1. MPMD Receiver Block Diagram

As shown in Fig.1 the receiver employs autocorrelation technique by using multiple delayed versions of the received signal. Then a bank of matched filters matched to ACF samples $\left(\boldsymbol{?}_{k}^{(n)}\right)$ of transmitted pulses for each user followed by a hard decision block separates the channels. Note that $D_{n}$ and $d_{n}$ are unique for each receiver channel. The output of the receiver is

$$
\mathbf{Y}=\operatorname{sgn}(\underbrace{\boldsymbol{P}_{k}^{(n)} \cdot \hat{\mathbf{r}}_{k, m}^{(n)}})
$$

Where

$$
\begin{array}{r}
\boldsymbol{?}_{k}^{(n)}=\left[\begin{array}{llll}
R_{p_{n} p_{n}}^{(n)}(1) & . & \cdot & R_{p_{n} p_{n}}^{(n)}(K)
\end{array}\right] \\
\hat{\mathbf{r}}_{k, m}^{(n)}=\left[\begin{array}{ccccc}
\hat{r}_{(1,1)}^{(n)} & \cdot & \cdot & \cdot & \hat{r}_{(1, m)}^{(n)} \\
\cdot & \cdot & & \cdot \\
\cdot & & \cdot & \cdot \\
\cdot & & \cdot & \cdot \\
\hat{r}_{(K, 1)}^{(n)} & \cdot & \cdot & \cdot & \hat{r}_{(K, m)}^{(n)}
\end{array}\right]
\end{array}
$$

$\lambda_{\mathrm{k}}^{(\mathrm{n})}$ denotes a vector of $K$ sampled values of the $\mathrm{n}^{\text {th }}$ user's transmitted pulses' normalized autocorrelation function. For instance $R_{p_{n}{ }^{p_{n}}}^{(n)}(1)$ represents the first sampling point (lag=0) of the normalized ACF for $\mathrm{n}^{\text {th }}$ user's transmitted pulse. Similarly, 
$\hat{\mathbf{r}}_{k, m}^{(n)}$ provides the sampled ACF matrix of $\mathrm{n}^{\text {th }}$ user's $\mathrm{m}^{\text {th }}$ bit for $K$ lags at the receiver. Each row of the matrix in (7) represents the correlation between the received signal and its delayed version based on various delays as shown in (8).

$$
\hat{r}_{k, m}^{n}=\int_{(m-1) T}^{(m-1) T+T_{i n}} r(t) \cdot r\left(t-\Delta_{n}\right) d t
$$

Where $T_{\text {in }}$ is the integration time that is most effective at pulse width and $\Delta_{n}$ is the total delay as

$$
\Delta_{n}=D_{n}+d_{n k}
$$

$D_{n}$ represents the $n^{\text {th }}$ users main delay representing lag zero in the ACF. $d_{n k}$ denotes the offset from its main delay or lag $k$ in $\mathrm{ACF}$ and its value is zero for $k=1\left(d_{n l}=0\right)$. Delaying the received signal by $D_{n}$ causes the "ref" pulse to align with the "data" pulse in each symbol where their product decodes the symbols by capturing the energy in lag zero of the generated ACF. Integrating this product over a finite time, samples the $\mathrm{ACF}$ at lag zero. Further delaying the received signal by multiple offsets $\left(d_{n k}\right)$ added to the main delay $\left(D_{n}\right)$ and multiplying with its un-delayed version, samples the autocorrelation function in non-zero lags after integration. The sampled points are estimates of the received signals' ACF. These estimated values $\left(\hat{\mathbf{r}}_{k, m}^{(n)}\right)$ are then matched to the original pulses sampled ACF values $\left(\boldsymbol{?}_{k}^{(n)}\right)$ and provide a more accurate decoding of the received symbols [6]. The MPMD method provides high performance in multipath channels. This is because the "ref" pulse and "data" pulse are correlated to each other, and the multipath channel introduces a longer duration in the signal component of the received signal, thus increasing the SNR at the output of the integrator. The high performance in multipath channels can be theoretically shown by considering a simpler case of (8) for one user transmitting one bit with sampling at lag-zero $(n=1, m=1, k=1)$ in a multipath channel as

$$
\begin{gathered}
\hat{r}=\int d t \sum_{l=1}^{L} \sum_{j=1}^{L} a_{l} \cdot a_{j} r\left(t-\tau_{l}\right) \cdot r\left(t-\tau_{j}-D\right) \\
\hat{r}=\sum_{l=1}^{L} \sum_{j=1}^{L} a_{l} \cdot a_{j} \cdot \underbrace{r\left(t-\tau_{l}\right) \cdot r\left(t-\tau_{j}-D\right)}_{R_{p p}\left(\tau_{l}-\tau_{j}-D\right)} d t \\
E\{\hat{r}\}=E\left\{L \cdot a_{l}^{2} R_{p p}(D)+\sum_{l=1}^{L} \sum_{j=1}^{L} a_{l} \cdot a_{j} \cdot R_{p p}\left(\tau_{l}-\tau_{j}-D\right)\right\}
\end{gathered}
$$

$R_{p p}(D)$ is equal to $R_{p p}(O)$ or the pulse energy $\left(E_{p}\right)$, since shifting the received signal by the main delay $(D)$, aligns the "ref" pulse with "data" pulse and samples lag-zero.

$$
E\{\hat{r}\}=L \cdot a_{l}^{2} \cdot E_{p}+\sum_{l=1}^{L} \sum_{j=1}^{L} R_{p p}\left(\tau_{l}-\tau_{j}-D\right) \cdot E\left\{a_{l} a_{j}\right\}
$$

$$
l \neq j
$$

Assuming that the received ray amplitudes $\left(a_{l}\right.$ and $a_{j}$ ) are uncorrelated, it is evident from the first term in (13) that the energy of the pulse will increase as the number of multipath rays $(L)$ increases.

\section{INDOOR CHANNEL MODEL}

The multipath model for the indoor radio channel used in this paper is the modified Saleh-Valenzuela (S-V) model proposed by Foerester and Li [3]. According to S-V model, multiple rays of the received signal arrive as clusters in an indoor channel, where the rays within each cluster are formed by multiple scatterers and reflectors that are closely spaced from each other [2]. The arrival times of clusters as well as the rays inside each cluster follow Poisson distribution while the received ray amplitudes follow Rayleigh distribution model. In this model the first cluster of rays represents arrival of paths close to LOS from transmitter to receiver and is usually formed by open spaces. The multipath indoor channel model proposed by Foerester and $\mathrm{Li}$ also demonstrates the clustering effect of $\mathrm{S}-\mathrm{V}$ model. However, a lognormal distribution is recommended for the received ray amplitudes instead of Rayleigh distribution. Fig. 2, represents the indoor multipath channel realization for a 2 user multiple access system.
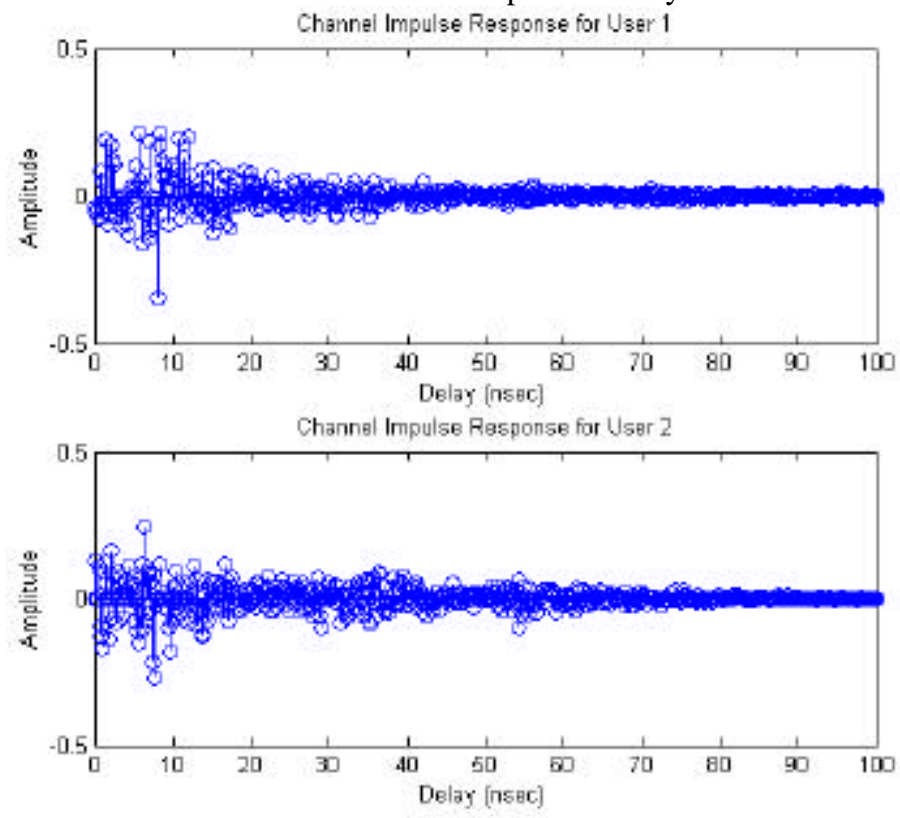

Figure 2. Channel Impulse Response for a 2-user MA Channel Based on Foerester-Li Model

\section{INDOOR CHANNEL PERFORMANCE}

In this section the performance of a MPMD based UWB system in a 2-user indoor channel is analyzed based on multiple delays and multiple autocorrelation sampling points (lags). All bit error rate simulations in this section were carried 
out with total number of 100,000 bits per data point. Fig. 3 represents the BER versus SNR for two users with only $1 \mathrm{nsec}$ time separation between them (i.e, $\mathrm{D}_{2}-\mathrm{D}_{1}=1 \mathrm{nsec}$ ) with multiple ACF sampling points $(K)$ at various SNR levels.

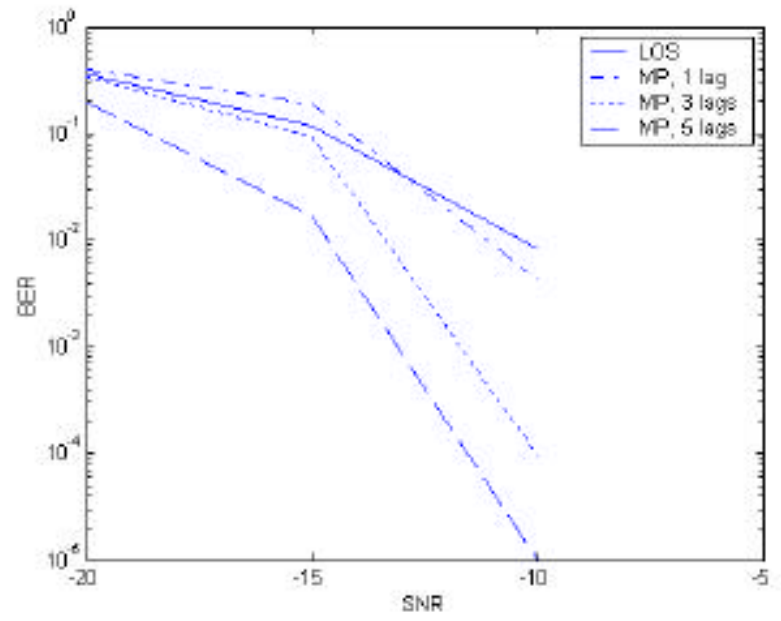

Figure 3. BER Versus Autocorrelation Sampling Points (K) for 2 Users at with 1ns Separation Between Users

As shown in this figure, the BER performance at $K=1$ in multipath case is close to non-multipath (LOS) case at lower signal to noise ratios. The multipath case outperforms the nonmultipath case at higher SNRs since the "ref" and "data" pulses are correlated to each other, and the multipath channel introduces a longer duration in the signal component of the received signal, thus increasing the overall signal energy at the output of the integrator. Fig. 3 shows a significant improvement in BER performance in a multipath channel when the number of ACF sampling points are increased. In other words, the multipath components in the received signal contain significant energy and the ability to capture that energy by sampling multiple points in the received signals $\mathrm{ACF}$ is quite important in low-power UWB communications systems. It is important to note that in order to have high performance in multipath channels, the pulses should be designed to be as orthogonal as possible with respect to shift. Otherwise, orthogonality may not be maintained with multipath and might result in lower performance.

\section{CONCLUSION}

The performance of MPMD multiple access method was evaluated in an indoor multipath channel. The channel model used for simulations in this paper is the modified S-V channel proposed by Foerester-Li from Intel. The MPMD method provides significant improvement in bit error rate performance of a UWB multiple access system in dense multipath channels. The analysis reveals that the use of multiple uncorrelated pulses minimizes the MAI and allows the system to increase the transmission rate. We also showed that the number of sampling points of the autocorrelation function plays an important role to increase the performance of a UWB multiple access system in multipath environments. The simulation results show that the proposed method is efficient as a multiple access UWB technique in dense multipath environments since it exploits the multipath phenomenon rather than suppressing it. Although these results are preliminary, we strongly believe that the MPMD approach is an efficient modulation scheme for multiple access UWB systems in indoor wireless channels.

\section{REFERENCES}

[1] www.fcc.gov

[2] A. A. M Saleh, R. A. Valenzuela, "A Statistical model for indoor multipath propagation”, IEEE JSAC, vol. SAC-5, No. 2, pp. 128-137, Feb, 1987.

[3] J. Foerster, Q.Li, "UWB Channel Modeling Contribution from Intel," IEEE P802.15-02/279-SG3a.

[4] H. Hashemi, "Impulse Response Modeling of Indoor Radio Propagation Channels," IEEE SJAC, vol.11, No. 7, pp. 967-978, Spet. 1993.

[5] F. Nekoogar, F. Dowla, "Multiple Access in Ultra-Wideband Communications Using Multiple Pulses", Wireless Data Communications Onboard Spacecraft-Technology and Applications Workshop” ESA/ESTEC, Noordwijk, NL, April 14-16, 2003.

[6] F. Nekoogar, F. Dowla, "On the Performance of Multiple Pulse Multiple Delay UWB Modulation, Wireless 2003, The Fifteenth International Conference on Wireless Communications, Calgary, Alberta Canada, July 7-9, 2003.

[7] A. Spiridon, F. Dowla, T. Rosenbury, and D. Benzel, “ Performance of Ultra-Wideband Differential Pulse Waveform”, Internal publication at Lawrence Livermore National Laboratory, Feb. 2001.

[8] R. Hoctor, H. Tomlinson, "Delay-hopped TransmittedReference RF Communications", 2002 IEEE Conference on Ultra Wideband Systems and Technologies, May 2002.

[9] M. Ghavami, L. B. Michael, R. Kohono, "Hermite Function Based Orthogonal Pulses for Ultra Wideband Communications", 2002 IEEE Conference on Ultra Wideband Systems and Technologies, May 2002.

[10] M.Z. Win and R. A. Scholtz, “ Ultra-wide Bandwidth TimeHopping Spread Spectrum Impulse Radio for Wireless Multiple Access Communications", IEEE Transactions on Communications, vol. 48, pp., 679-691, Apr. 2000.

[11] C. J. LeMarttret, G. B. Giannkis, “All-digital PAM Impulse radio for multiple-access through frequency-selective multipath" Global Telecommunications Conference, 2000. GLOBECOM '00.

[12] J.D. Choi, W. E. Stark, "Performance of Autocorrelation Receivers for Ultra-Wideband communications with PPM in Multipath Channels", IEEE Conference on Ultra Wideband Systems and Technologies, 2002.

[13] M.R. Winkler, "Chirp Signals for Communication", IEEE Wescon Conc. 1962.

[14] G. F. Gott and J. P. Newsome, "H.F. Data Transmission Using Chirp Signals”, Proc. of IEE., vol. 118, pp. 1162-1166, Sept. 1971. 
[15] S.E.El-Khamy, S.E.Shaaban, E.A.Thabet, "Multiuser Chirp Modulation Signals (M-CM0 for Efficient Multiple Access Communication Systems", Radio Science Conference, March 1996.

[16] J. G. Proakis, Digital Communications, Third Eddition, McGraw-Hill, Inc., New York, 1995

[17] ] Y. K. Jeong, T. K. Song, "Simultaneous Multizone Focusing Method with Orthogonal Chirp Signals", IEEE Ultrasonics Symposium Proceedings, 2001.

[18] F. Zhu, Z. Wu, C. R. Nassar, " Generalized Fading Channel Model With Application to UWB", IEEE Conference on Ultra Wideband Systems and Technologies, 2002. 